Original Research Paper

\title{
Effect of Pre-Slaughter Autophagic Status on Postmortem Proteolysis in Skeletal Muscle of Mice
}

\author{
${ }^{1}$ Tomonori Nakanishi, ${ }^{1}$ Miho Yamashita, ${ }^{1}$ Naoto Nishimoto, ${ }^{2}$ Laurie Erickson and ${ }^{1}$ Satoshi Kawahara \\ ${ }^{I}$ Department of Biochemistry and Applied Biosciences, Faculty of Agriculture, \\ University of Miyazaki, 1-1 Gakuenkibanadai-nishi, Miyazaki 889-2192, Japan \\ ${ }^{2}$ Department of Biology, Harold Washington City College of Chicago, 30 E. Lake St, Chicago IL 60601, USA
}

Article history

Received: 06-06-2017

Revised: 29-06-2017

Accepted: 08-07-2017

Corresponding Author:

Satoshi Kawahara

Department of Biochemistry

and Applied Biosciences,

Faculty of Agriculture,

University of Miyazaki, 1-1

Gakuenkibanadai-nishi,

Miyazaki 889-2192, Japan

Tel: +81985587204

Fax: +81985587204

E-mail: a04206u@cc.miyazaki-u.ac.jp

\begin{abstract}
Post mortem proteolysis is a key event during the aging process, where muscle converts to meat. However, its precise mechanism remains unclear. In this study, the effects of pre-slaughter autophagic status on post mortem proteolysis were investigated using mouse skeletal muscle. Mice were fasted for $14 \mathrm{~h}$ and administered with or without the autophagy inhibitor wortmannin, whereas control mice were given free access to diet. Skeletal muscle samples were collected and stored at $4^{\circ} \mathrm{C}$. Muscle proteins were extracted at several time points, followed by evaluation of microtubule-associated protein Light Chain 3 (LC3)-II expression as an autophagic marker and of post mortem changes in water-soluble proteins by High Performance Liquid Chromatography (HPLC) analysis. Mice fasted before sacrifice showed significantly higher expression of LC3-II than control mice and this effect was inhibited by the administration of wortmannin. Results on HPLC analysis indicated typical post mortem changes in water-soluble muscle proteins and these changes were accelerated when autophagy was induced by starvation. In addition, the administration of wortmannin canceled acceleration of post mortem changes by starvation. These findings could suggest that autophagy is a mediator of post mortem proteolysis in skeletal muscle and contributes to meat quality.
\end{abstract}

Key words: Autophagy, Post Mortem Proteolysis, Mouse, Skeletal Muscle

\section{Introduction}

Skeletal muscle of domestic animals converts to meat through the aging process in which the fresh carcass or skeletal muscle is kept at low temperature for a certain period of time. During the meat aging process, structural changes in myofibrils are observed, as indicated by weakening of Z-disk (Takahashi and Hattori, 1989), dissociation of bonds between actin and myosin filaments formed as a result of rigor mortis (Takahashi et al., 1967) and fragmentation of connectin and nebulin filaments (Takahashi, 1996). Previous studies have also reported significant proteolysis not only of miofiblillar proteins but also of water-soluble sarcoplasmic proteins during the aging process (Bowker et al., 2012). These proteolytic events are considered to improve meat quality traits such as tenderness and flavor (Jelenikova et al., 2008; Dashdorj et al., 2015).

A number of studies have addressed the mechanism by which the aging process improves meat quality traits.
Some of these studies have pointed out an important role of $\mathrm{Ca}^{2+}$-dependent neutral proteases, calpains, on meat aging and $\mu$-calpain is considered to exert the primary activity in post mortem tenderization among calpain isoforms (Koohmaraie and Geesink, 2006). Several members of the cathepsin family, which are mainly lysosomal enzymes and have both exo- and endopeptidase activity, have also been shown to be involved in reactions during post mortem aging (Kitamura et al., 2010). However, post mortem changes in skeletal muscle are comprised of complex chemical and biological systems and the precise mechanism involved in proteolysis and improvement of meat quality during the aging process is not fully understood.

Autophagy is the bulk degradation process for intracellular components in eukaryotic cells and takes the central role in the recycling system for proteins and organelles to maintain the nutrient supply under conditions of starvation and other stresses (Mizushima and Komatsu, 2011). Autophagy can be classified into three types: Macro 
autophagy, micro autophagy and chaperone-mediated autophagy (Mizushima et al., 2008). Among them, macro autophagy (simply referred to as autophagy hereafter) plays the leading role in biological regulation (Mizushima et al., 2010) and has been better studied in the case of skeletal muscle (Sandri et al., 2013). Since autophagy is responsible for the turnover of cell components not only in response to a variety of stresses but also under constitutive conditions, the abnormal regulation of autophagy can cause detrimental effects on myofibrils, leading to human muscle diseases such as autophagic vacuolar myopathies and muscular dystrophies (Sandri et al., 2013). Furthermore, deletion of the autophagy-related gene $\operatorname{Atg} 7$ in mice resulted in muscle atrophy and decreased muscle force (Masiero et al., 2009).

These findings strongly suggest that autophagy is an essential metabolic pathway to control muscle homeostasis and maintain muscle mass and functions. The questions of whether autophagy modulates post mortem changes in skeletal muscle and affects meat quality have not been answered. Here, we investigated the effects of pre-slaughter autophagic status on post mortem changes in mouse skeletal muscle proteins, to address the potential involvement of autophagy in meat quality.

\section{Materials and Methods}

\section{Animals and Treatments}

Adult female C57BL/6 mice were purchased from Japan SLC, Inc. (Shizuoka, Japan). Animals were used in accordance with the guidelines for the care and use of laboratory animals at the University of Miyazaki and Law No.105 of the Japanese government. The experimental design for the present study was approved by the University of Miyazaki (approval number: 2014001 ). Mice were kept at $25^{\circ} \mathrm{C}$ on a $12 \mathrm{~h}$ dark- $12 \mathrm{~h}$ light cycle with free access to water and a commercial diet (Labo MR stock, Nosan Corporation, Yokohama, Japan) until initiation of experiments. Mice aged eight weeks were randomly assigned to groups of three mice each. Two experimental groups were deprived of a diet and were intraperitoneally administered with $500 \mu \mathrm{L}^{-1}$ of saline containing 2\% Dimethyl Sulphoxide (DMSO) or $0.2 \mathrm{~mm}$ wortmannin dissolved in DMSO. The control mice were intraperitoneally administered with saline containing $2 \%$ DMSO and given free access to diet. Mice were sacrificed under anesthesia with inhaled isoflurane $14 \mathrm{~h}$ after the start of fasting and intraperitoneal administration and skeletal muscles were collected from the femoral area.

\section{Sample Preparation}

The skeletal muscles were stored at $4^{\circ} \mathrm{C}$. Skeletal muscle samples weighing about $70 \mathrm{mg}$ were excised 0 , $8,24,48$ and $96 \mathrm{~h}$ after sacrifice of mice and water- soluble proteins were extracted from each sample. Briefly, skeletal muscle samples were homogenized in 1 $\mathrm{mL}$ of water containing protease inhibitors (Complete Mini, Roche, Indianapolis, IN, USA). After centrifugation at $9400 \mathrm{rpm}$ for $15 \mathrm{~min}$, the supernatant was mixed with Sodium Dodecyl Sulfate (SDS) sample buffer consisting of $63 \mathrm{mM}$ tris- $\mathrm{HCl}$ (pH 6.8), 2\% SDS, $5 \%$ sucrose, $0.005 \%$ bromophenol blue and 5\% 2mercaptoethanol and then heated at $95^{\circ} \mathrm{C}$ for $5 \mathrm{~min}$. After each sample of protein extract was diluted so that SDS concentration was less than $0.1 \%$, the protein concentration was determined by the Bradford assay. All samples were kept at $-20^{\circ} \mathrm{C}$ until used.

\section{Western Blotting Analysis}

Protein extracts of skeletal muscles excised immediately after sacrifice were used for detection of microtubule-associated protein Light Chain 3 (LC3). Equal amounts of protein were separated by electrophoresis on $15 \%$ SDS-polyacrylamide gels (SuperSep Ace, Wako Pure Chemical Industries, Ltd., Osaka, Japan). After electrophoresis, the proteins were transferred onto polyvinylidene fluoride membrane. The membrane was then blocked by $10 \%$ skim milk in Tris-buffered saline followed by each antibody binding reaction. The rabbit anti-mouse LC3 antibody was purchased from MBL Laboratories (Nagoya, Japan). After the secondary antibody reaction by goat anti-rabbit IgG conjugated with horseradish peroxidase (Santa Cruz Biotechnology, Santa Cruz, CA, USA), detection of each protein was performed using an ImmunoStar reagent (Wako Pure Chemical Industries). Luminescence was quantified by a ChemiDoc system (Bio-Rad Laboratories, Hercules, CA, USA).

\section{High Performance Liquid Chromatography (HPLC) Analysis}

The fraction of water-soluble proteins was analyzed using a gel filtration HPLC method. Each extracted sample was diluted by the mobile phase, $50 \mathrm{~mm}$ phosphate buffer ( $\mathrm{pH} 7.0$ ) containing $100 \mathrm{mM} \mathrm{NaCl}$, to adjust protein concentration to $1 \mathrm{mg} \mathrm{Ml}{ }^{-1}$. After passing through a $0.45 \mu \mathrm{m}$ filter, $20 \mu \mathrm{g}$ of each protein sample was analyzed using a system composed of a degasser (FRC-10A, Shimadzu Corporation, Kyoto, Japan), two pumps (LC-10AD, Shimadzu Corporation), a column oven (CTO-20A, Shimadzu Corporation), a UV detector (SPD-20A, Shimadzu Corporation) and a gel filtration column (Shodex PROTEIN KW-803, Showa denko, Tokyo, Japan). Temperature was set at $40^{\circ} \mathrm{C}$ and detection was carried out at $220 \mathrm{~nm}$ with a flow rate of $0.2 \mathrm{~mL} / \mathrm{min}$.

\section{Statistical Analyses}

All analyses were performed using the GraphPad Prism version 6.0 (GraphPad Software, La Jolla, CA, 
USA). Differences in band intensities on western blotting analysis among groups were compared using one-way Analysis of Variance (ANOVA) followed by the Tukey's Multiple Comparison test. Each peak area calculated in HPLC analysis was analyzed by comparing groups using a two-way ANOVA (factors: Treatment group and postmortem time). Tukey's multiple comparison analysis was carried out to compare treatment groups at each time point when significant interaction was observed in a two-way ANOVA. Statistical significance was defined as $\mathrm{p}<0.05$.

\section{Results}

\section{Western Blotting Analysis for Detection of LC3 Expression}

To evaluate the degree of autophagy in skeletal muscle at the time of sacrifice, LC3 expression was detected by western blotting analysis. Our results showed that the expression of LC3-II, the phosphatidyl ethanolamine-conjugated form, was up regulated when mice were deprived of a diet before sacrifice (Fig. 1A). Relative expression of LC3-II to LC3-I in mice starved for $14 \mathrm{~h}$ was significantly increased compared to that in control mice given free access to diet, showing autophagy induction (Fig. 1B). Although autophagy induction was also observed under administration of wortmannin, the calculated ratio of LC3-II to LC3-I in this group was $54 \%$ lower than that of mice deprived of a diet and administered saline (Fig. 1B).

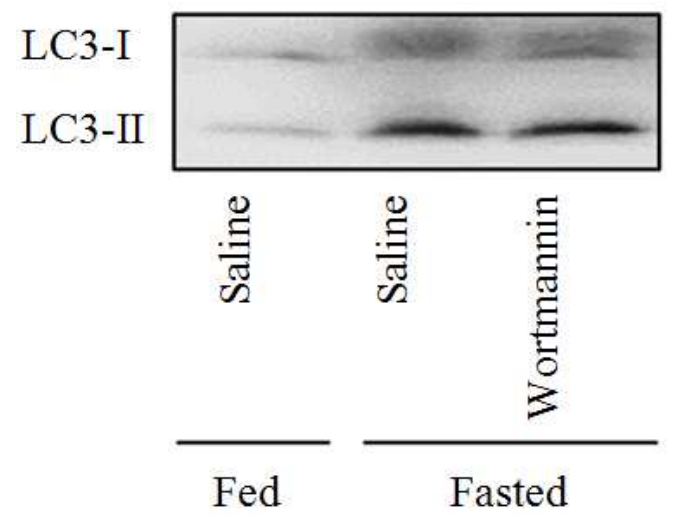

(A)

\section{Postmortem Changes in Water-Soluble Proteins}

HPLC analysis was performed to evaluate postmortem changes in the fraction of water-soluble proteins. A comparison of the chromatogram between samples prepared $0 \mathrm{~h}$ and $96 \mathrm{~h}$ after sacrifice in control mice indicated the postmortem changes in water-soluble proteins (Fig. 2A). The peak area of fractions with a retention time of 17.5-20 min (peak 1) and 30-34 $\mathrm{min}$ (peak 3) was increased over time, whereas that of the fraction with retention time of 27-30 min (peak 2) was decreased. The area percent of these three fractions was analyzed to assess the degree of post mortem change in each treatment group. Our results showed that $14 \mathrm{~h}$ starvation significantly accelerated the increase in the area percent of peak 1 throughout the postmortem period in mice administered saline (Fig. 2B). However, the effect of starvation on peak 1 was canceled by intraperitoneal administration of wortmannin. The results also showed that mice fasted and administered saline exhibited the lowest area percent of peak 2 among groups and that wortmannin inhibited the starvationinduced decrease in peak 2 , albeit a significant difference was observed only at $48 \mathrm{~h}$ after euthanasia (Fig. 2C). In contrast, there were no significant differences in area percent of peak 3 among groups (Fig. 2D). In this study, correlational analyses were also performed to clarify the relationship between pre-slaughter autophagic status of each mouse (the LC3-II/LC3-I ratio) and subsequent proteolysis (the area percent of each peak). Our results indicated that pre-slaughter autophagic status correlated positively with the area percent of peak 1 and negatively with that of peak 2 (Table 1).

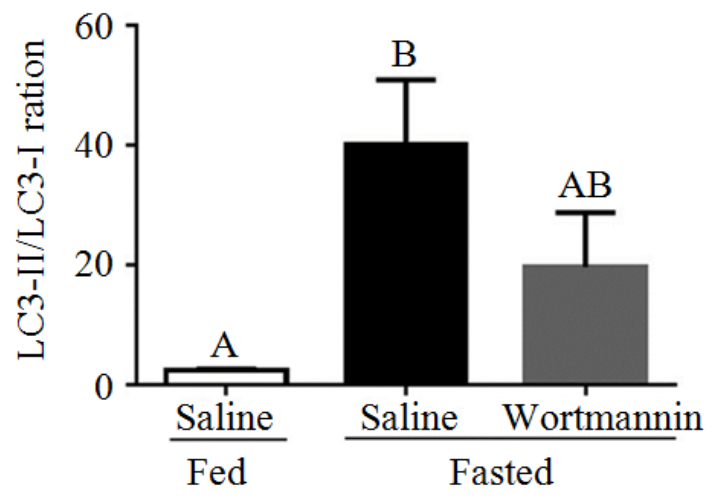

(B)

Fig. 1. Effect of starvation and administration of wortmannin on microtubule-associated protein Light Chain 3 (LC3) expression in mouse skeletal muscle. Mice were fasted for $14 \mathrm{~h}$ and administered with or without an autophagy inhibitor wortmannin, whereas control mice were given free access to diet. Skeletal muscle proteins were extracted immediately after sacrifice and equal amounts of proteins were resolved by SDS-PAGE followed by western blotting with antibodies specific for LC3. (A) Representative band patterns of each experimental group. (B) Differences in relative band intensities of LC3-II to LC3-I among groups were compared using one-way analysis of variance followed by the Tukey's Multiple Comparison test. Data are expressed as means \pm S.E.M. Groups with different letters are significantly different $(p<0.05)$. 


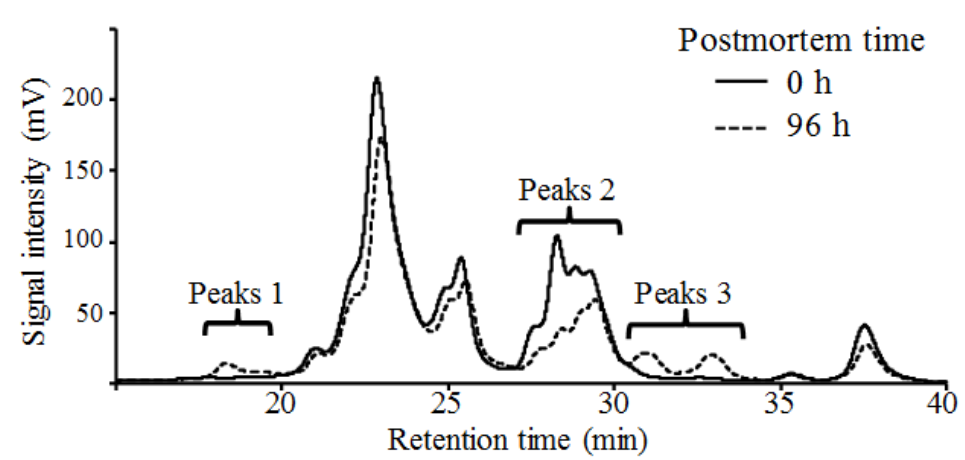

(A)

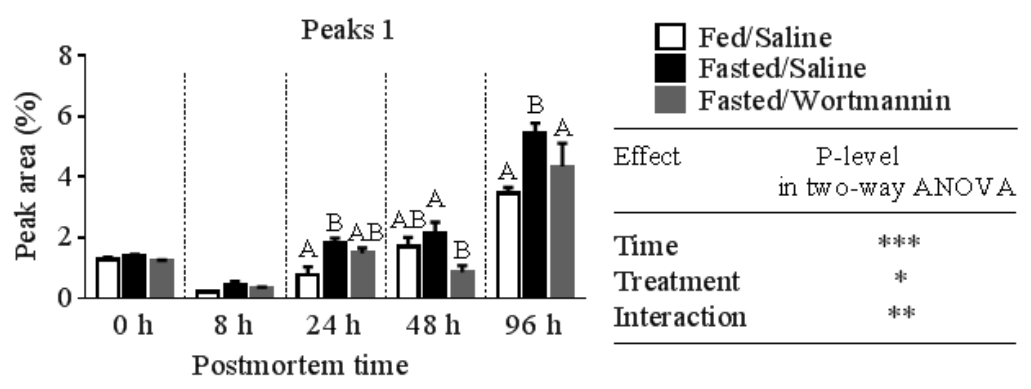

(B)
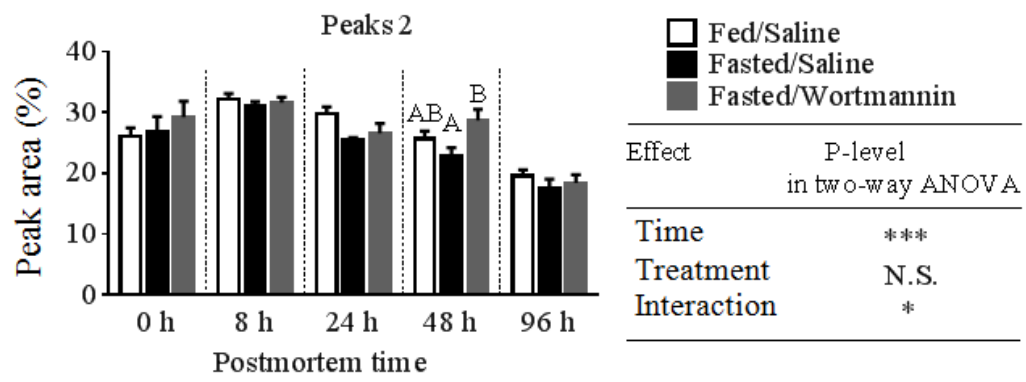

(C)

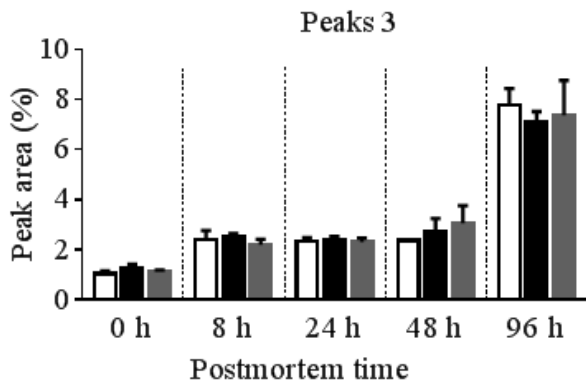

\begin{tabular}{|c|c|}
\hline Effect & $\begin{array}{c}\text { P-level } \\
\text { in two-way ANOVA }\end{array}$ \\
\hline Time & 水水水 \\
\hline Treatment & N.S. \\
\hline Interaction & N.S. \\
\hline
\end{tabular}

(D)

Fig. 2. Effect of starvation and administration of wortmannin on post mortem changes in water-soluble proteins. Skeletal muscle samples of each group were kept at $4{ }^{\circ} \mathrm{C}$ and water-soluble proteins were prepared at different time points. Equal amounts of proteins were resolved and analyzed by gel filtration HPLC analysis; (A) Comparison of typical chromatograms between protein extracts prepared $0 \mathrm{~h}$ and $96 \mathrm{~h}$ after sacrifice in the control group; (B-D) Each peak area calculated in HPLC analysis was analyzed by comparing groups using a two-way ANOVA (factors: Treatment group and postmortem time). Tukey's multiple comparison analysis was carried out to compare treatment groups at each time point when significant interaction was observed in the two-way ANOVA. Data are expressed as means \pm S.E.M. Groups with different letters are significantly different $(p<0.05)$ 
Table 1. Correlations between pre-slaughter autophagic status (LC3-II/LC3-I ratio) and peak areas of the HPLC fractions

\begin{tabular}{llll}
\hline Peaks on HPLC & Post mortem time & Pearson's coefficient correlation & P level \\
\hline 1 & $0 \mathrm{~h}$ & 0.56490 & N.S. \\
& $8 \mathrm{~h}$ & 0.91250 & $* *$ \\
& $24 \mathrm{~h}$ & 0.76520 & N.S \\
& $48 \mathrm{~h}$ & 0.38520 & $*$ \\
& $96 \mathrm{~h}$ & 0.71000 & N.S. \\
2 & $0 \mathrm{~h}$ & -0.1956 & N.S. \\
& $8 \mathrm{~h}$ & -0.2923 & $*$ \\
& $24 \mathrm{~h}$ & -0.7152 & N.S. \\
& $48 \mathrm{~h}$ & -0.5469 & N.S. \\
& $96 \mathrm{~h}$ & -0.5653 & N.S. \\
3 & $0 \mathrm{~h}$ & 0.46680 & N.S. \\
& $8 \mathrm{~h}$ & -0.0158 & N.S. \\
& $24 \mathrm{~h}$ & -0.1147 & N.S. \\
$48 \mathrm{~h}$ & -0.2020 & N.S. \\
\hline
\end{tabular}

\section{Discussion}

LC3 is essential to the progression of autophagy in mammals and the level of conversion of LC3-I to LC3-II is an indicator of autophagic activity (Kabeya et al., 2000; Karim et al., 2007). In addition, LC3 is the unique autophagy-related gene that can be controlled by nutrient supply and deprivation (Kadowaki et al., 2006). Therefore, we investigated the relative expression of LC3-II to LC3-I as an index of autophagic status in skeletal muscle at the time of sacrifice. Our results clearly showed that 14 h-starvation induced autophagy in skeletal muscle (Fig. 1). Wortmannin is a well-known selective inhibitor of phosphatidylinositol 3-kinase whose activity is required for autophagy (Blommaart et al., 1997). The inhibitory effect of wortmannin on starvation-induced autophagy seemed to be confirmed in this study, indicated by decreased relative expression levels of LC3II to LC3-I (Fig. 1). Our preliminary experiments showed that wortmannin failed to decrease the LC3II/LC3-I ratio in fed mice because the expression level of LC3-II was quite low under fed conditions, regardless of the presence or absence of wortmannin (data not shown). Therefore, the group of mice given free access to a diet and administered wortmannin was excluded in this study and further experiments were carried out by using the above three groups of mice.

In this study, we investigated the effects of preslaughter administration of wortmannin on post mortem changes in muscle proteins using HPLC analysis. Previous studies have reported significant proteolysis of water-soluble proteins as well as myofibrillar proteins during the aging process of meat (Bowker et al., 2012). Although water-soluble sarcoplasmic proteins are not structural proteins in skeletal muscle, their post mortem proteolysis has been shown to occur in parallel with tenderization of meat (Bowker et al., 2008). Moreover, the water-soluble fraction contains fragments of myofibrillar proteins, which become extractable by post mortem proteolysis (Di Luca et al., 2013). Therefore, post mortem changes in water-soluble proteins are considered useful indicators of meat tenderness. Results from this study with HPLC analysis also showed post mortem time-dependent changes in water-soluble proteins, as indicated by increased areas of peaks 1 and 3 and a decreased area of peak 2 (Fig. 2). Peaks 1 and 2 seemed to exhibit bell-shaped time responses where the area percent of peak 1 decreased at the early time point $(8 \mathrm{~h})$ but increased thereafter and that of peak 2 increased $8 \mathrm{~h}$ after sacrifice but decreased subsequently. Both rigor mortis and its resolution are well known to affect solubility of a wide range of muscle proteins (Sayre et al., 1963). Given that rigor mortis develops within several hours in mice (Annesley and Walker, 1980), a low amount of peak 1 and a high amount of peak 2 observed at $8 \mathrm{~h}$ could be attributed to rigor mortis-mediated changes in solubility of these proteins. In this study, skeletal muscle of mice in which autophagy was induced by starvation before sacrifice exhibited significantly greater changes in proteins indicated by peak 1 than that of control mice. Mice fasted and administered saline exhibited slightly lower area percent of peak 2 than control mice at least $48 \mathrm{~h}$ after sacrifice, although there was no difference in peak 3 between these two groups. In addition, the effects of starvation on post mortem changes in peaks 1 and 2 seemed to be inhibited in mice administered wortmannin. Furthermore, the present study showed that there were high correlations between the LC3-II/LC3-I ratio of each mouse at the time of sacrifice and postmortem changes in muscle proteins (Table 1). These findings suggest that preslaughter autophagic status affects proteolytic degradation of some sarcoplasmic or myofibrillar proteins during the post mortem period.

The aging process is well-known to increase meat quality since the beginning of the last century (Koohmaraie, 1994). However, the mechanisms involved in the aging process is still poor understood, due to its complex features of pre-slaughter and post-slaughter 
factors. Some studies have elucidated that activities of endogenous enzymes including calpains and cathepsins play important roles on proteolysis of myofibrillar proteins and on meat quality (Kitamura et al., 2010; Koohmaraie and Geesink, 2006), whereas others have demonstrated that several non-enzymatic aspects such as temperature, $\mathrm{pH}$ and $\mathrm{Ca}^{2+}$ concentration affect meat quality traits, especially tenderness (Takahashi, 1996). More recently, studies using beef and chicken muscle have shown that apoptotic factors such as caspase-3 are upregulated during the early post mortem period, suggesting potential involvement of apoptosis in proteolytic degradation of muscle proteins (Che et al., 2011; Huang et al., 2016). Given that proteolysis is the most characteristic biochemical event during the aging process, two major protein degradation machineries in cells, the ubiquitin proteasome system and the autophagy system, could also be involved in meat quality. Indeed, the $20 \mathrm{~S}$ proteasome, but not the $26 \mathrm{~S}$ proteasome, was reported to have effects on tenderization of rabbit meat during aging as well as under high pressure treatment (Otsuka et al., 1998). Lamare et al. (2002) also indicated a potential role of proteasome in beef tenderization by showing its stable post mortem activity.

The hypothesis that proteolytic degradation of muscle proteins is controlled by autophagy, at least in combination with other systems should be considered reasonable given that endo-lysosomal proteases, including cathepsins, play important roles in the degradation system of autophagy (Mizushima et al., 2008) and that cytoplasmic $\mathrm{Ca}^{2+}$ is known to be an inducer of autophagy (Weiss and Minke, 2015). However, there is little information concerning the involvement of autophagy on post mortem proteolysis. Garcia-Macia et al. (2014) showed that the expression level of beclin 1, an essential regulator of autophagy, was increased transiently at the early post mortem period of beef aging and that there was a difference in post mortem expression of LC3 between two beef breeds. To the best of our knowledge, however, it remained unclear whether autophagy is actually implicated in degradation of muscle proteins during the aging process. This study is the first report that pre-slaughter autophagic status affects subsequent post mortem proteolysis of skeletal muscle. In this study, the LC3-II/LC3-I ratio in skeletal muscle was investigated as being one of most commonly used indexes of autophagic status. However, several reports have pointed out that in some cases conversion of LC3-I to LC3-II might be observed independently of autophagic activity (Hara et al., 2008; Matsunaga et al., 2009). Further studies are needed to elucidate whether autophagy is directly involved in meat quality and whether the results obtained in the present study using mouse skeletal muscle can be reproduced in edible meat.

Myofibrillar and cytoskeletal proteins including troponin T, troponin I, desmin, vinculin, meta-vinculin, dystrophin, nebulin and connectin, have been shown to be degraded during the post mortem proteolysis
(Koohmaraie and Geesink, 2006). In this study, we performed HPLC analysis to investigate overall changes in water-soluble proteins and have not determined whether autophagy directly accelerates degradation of myofibrillar and cytoskeletal proteins. The molecular identification of substrates which are degraded by autophagy during the postmortem period should be carried out in the future, to elucidate the direct involvement of autophagy in meat quality.

\section{Conclusion}

The present study demonstrated that autophagy induced by starvation before sacrifice accelerated subsequent post mortem changes in mouse skeletal muscle proteins. In addition, the administration of an autophagy inhibitor wortmannin partially canceled acceleration of post mortem changes by starvation. These findings could suggest that pre-slaughter autophagic status affects post mortem proteolysis and meat quality.

\section{Acknowledgment}

This study was supported by the Research Grant for Young Investigators of Faculty of Agriculture, University of Miyazaki.

\section{Author's Contributions}

Tomonori Nakanishi: Designed and performed experiments, analyzed data and drafted the manuscript.

Miho Yamashita: Performed experiments and analyzed the data.

Naoto Nishimoto: Performed experiments and analyzed the data.

Laurie Eriskson: Designed experiments and revised the manuscript critically for important intellectual content.

Satoshi Kawahara: Designed experiments and wrote the manuscript.

\section{Ethics}

All animal experiments were performed in accordance with the guidelines for the care and use of laboratory animals at the University of Miyazaki and Law No.105 of the Japanese government.

\section{Conflict of Interest}

The authors declare no conflict of interest.

\section{References}

Annesley, T.M. and J.B. Walker, 1980. Energy metabolism of skeletal muscle containing cyclocreatine phosphate. Delay in onset of rigor mortis and decreased glycogenolysis in response to ischemia or epinephrine. J. Biol. Chem., 255: 3924-3930. 
Blommaart, E.F., U. Krause, J.P. Schellens, H. Vreeling-Sindelarova and A.J. Meijer, 1997. The phosphatidylinositol 3-kinase inhibitors wortmannin and LY294002 inhibit autophagy in isolated rat hepatocytes. Eur. J. Biochem., 243: 240-246.

Bowker, B.C., T.M. Fahrenholz, E.W. Paroczay and M.B. Solomon, 2008. Effect of hydrodynamic pressure processing and aging on sarcoplasmic proteins of beef strip loins. J. Muscle Food, 19: 175-193.

Bowker, B.C., T.M. Fahrenholz, P.J. Sarnoski and M.B. Solomon, 2012. Alterations in the sarcoplasmic protein fraction of beef muscle with postmortem aging and hydrodynamic pressure processing. J. Food Sci., 77: C594-602.

Che, L., XC. Feng, F. Lu, X.L. Xu and G.H. Zhou 2011. Effects of camptothecin, etoposide and $\mathrm{Ca}^{2+}$ on caspase-3 activity and myofibrillar disruption of chicken during postmortem ageing. Meat Sci., 87: 165-174.

Dashdorj, D., T. Amna and I. Hwang, 2015. Influence of specific taste-active components on meat flavor as affected by intrinsic and extrinsic factors: An overview. Eur. Food Res. Technol., 241: 157-171.

Di Luca, A., G. Elia, A.M. Mullen and R.M. Hamill, 2013. Monitoring post mortem changes in porcine muscle through 2-D DIGE proteome analysis of Longissimus muscle exudate. Proteome Sci., 11: 9-9. DOI: $10.1186 / 1477-5956-11-9$

Garcia-Macia, M., V. Sierra, A. Palanca, I. Vega-Naredo and D. de Gonzalo-Calvo, 2014. Autophagy during beef aging. Autophagy, 10: 137-143.

Hara, T., A. Takamura, C. Kishi, S. Iemura and T. Natsume et al., 2008. FIP200, a ULK-interacting protein, is required for autophagosome formation in mammalian cells. J. Cell Biol., 181: 497-510.

Huang, F., M. Huang, H. Zhang, C. Zhang and D. Zhang et al., 2016. Changes in apoptotic factors and caspase activation pathways during the postmortem aging of beef muscle. Food Chem., 190: 110-114.

Jelenikova, J., P. Pipek and M. Miyahara, 2008. The effects of breed, sex, intramuscular fat and ultimate pH on pork tenderness. Eur. Food Res. Technol., 227: 989-994.

Kabeya, Y., N. Mizushima, T. Ueno, A. Yamamoto and T. Kirisako et al., 2000. $\mathrm{LC}_{3}$, a mammalian homologue of yeast Apg8p, is localized in autophagosome membranes after processing. EMBO J., 19: 5720-5728.

Kadowaki, M., M.R. Karim, A. Carpi and G. Miotto, 2006. Nutrient control of macroautophagy in mammalian cells. Mol. Aspects Med., 27: 426-443.

Karim, M.R., T. Kanazawa, Y. Daigaku, S. Fujimura and G. Miotto et al., 2007. Cytosolic $\mathrm{LC}_{3}$ ratio as a sensitive index of macroautophagy in isolated rat hepatocytes and H4-II-E cells. Autophagy, 3: 553-560.
Kitamura, S., K. Kudo, K. Chikuni, T. Watanabe and T. Nishimura, 2010. Actions of cathepsins on troponin $\mathrm{T}$ during postmortem aging of porcine muscle. Anim. Sci. J., 81: 501-505.

Koohmaraie, M., 1994. Muscle proteinases and meat aging. Meat Sci., 36: 93-104.

Koohmaraie, M. and G.H. Geesink, 2006. Contribution of postmortem muscle biochemistry to the delivery of consistent meat quality with particular focus on the calpain system. Meat Sci., 74: 34-43.

Lamare, M., R.G. Taylor, L. Farout, Y. Briand and M. Briand, 2002. Changes in proteasome activity during postmortem aging of bovine muscle. Meat Sci., 61: 199-204.

Masiero, E., L. Agatea, C. Mammucari, B. Blaauw and E. Loro et al., 2009. Autophagy is required to maintain muscle mass. Cell Metabol., 10: 507-515.

Matsunaga, K., T. Saitoh, K. Tabata, H. Omori and T. Satoh et al., 2009. Two beclin 1-binding proteins, Atg14L and Rubicon, reciprocally regulate autophagy at different stages. Nat. Cell Biol., 11: 385-396.

Mizushima, N. and M. Komatsu, 2011. Autophagy: Renovation of cells and tissues. Cell, 147: 728-741.

Mizushima, N., B. Levine, A.M. Cuervo and D.J. Klionsky, 2008. Autophagy fights disease through cellular self-digestion. Nature, 451: 1069-1075.

Mizushima, N., T. Yoshimori and B. Levine, 2010. Methods in mammalian autophagy research. Cell, 140: 313-326.

Otsuka, Y., N. Homma, K. Shiga, J. Ushikim and Y. Ikeuchi et al., 1998. Purification and properties of rabbit muscle proteasome and its effect on myofibrillar structure. Meat Sci., 49: 365-378.

Sandri, M., L. Coletto, P. Grumati and P. Bonaldo, 2013. Misregulation of autophagy and protein degradation systems in myopathies and muscular dystrophies. J. Cell Sci., 126: 5325-5333.

Sayre, R.N. and E.J. Briskey, 1963. Protein solubility as influenced by physiological conditions in the muscle. J. Food Sci., 28: 675-679.

Takahashi, K., 1996. Structural weakening of skeletal muscle tissue during post-mortem ageing of meat: The non-enzymatic mechanism of meat tenderization. Meat Sci., 43: 67-80.

Takahashi, K., T. Fukazawa and T. Yasui, 1967. Formation of myofibrillar fragments and reversible contraction of sarcomeres in chicken pectoral muscle. J. Food Sci., 32: 409-413.

Takahashi, K. and A. Hattori, 1989. $\alpha$-Actinin is a component of Z-filament, a structural backbone of skeletal muscle Z-disks. J. Biochem., 105: 529-536.

Weiss, S. and B. Minke, 2015. A new genetic model for calcium induced autophagy and ER-stress in drosophila photoreceptor cells. Channels, 9: 14-20. 